\title{
Erratum
}

\section{Adiabatic Theorems and Applications to the Quantum Hall Effect ${ }^{\star}$}

\author{
J. E. Avron ${ }^{1}$, R. Seiler ${ }^{2}$ and L. G. Yaffe ${ }^{3}$ \\ 1 Department of Physics, Technion, Haifa 32000, Israel \\ 2 Fachbereich Mathematik, Technische Universität Berlin, D-10623 Berlin, Germany \\ 3 Jadwin Hall, Princeton University, Princeton, NJ 08544, USA
}

Received February 8, 1993; in revised form March 22, 1993

Commun. Math. Phys. 110, 33-49 (1987)

After half a decade since the appearance of our article [1] we sill regularly receive questions and remarks about some formulas and expressions in text. Furthermore Dr. M. Hübner (Leipzig) points out that some term in Eqs. (2.12), (2.14), (2.16) vanishes. So we write this erratum:

1. On p. 37 in the formula just after Eq. (1.3) there is a minus sign missing:

$$
H_{A}(s, P)=+\left.(i / \tau)\left[\partial_{s} U_{A}\left(s ; s^{\prime}, P\right)\right] U_{A}^{*}\left(s ; s^{\prime}, P\right)\right|_{s=s^{\prime}} ;
$$

the last term in this expression is, of course, one.

2. On p. 37 last line: There is a minus sign missing; the formula should read

$$
H^{\prime}(s)[H(s)+i]^{-1}=-[H(s)+i] \partial_{s}[H(s)+i]^{-1} .
$$

3. Part (b) of Theorem 2.4 should read: For all $j \in \mathscr{N}$,

$$
\begin{array}{ll}
\Omega_{2 j-1}(s)=O\left(1 / \tau^{j}\right), & \Omega_{2 j}(s)=O\left(1 / \tau^{j}\right), \\
R_{2 j-1}(s)=O\left(1 / \tau^{j}\right), & R_{2 j}(s)=O\left(1 / \tau^{j}\right),
\end{array}
$$

where

$$
R_{n}(s):=\Omega(s)-\sum_{j=0}^{n-1} \Omega_{j}(s) .
$$

All bounds are uniform in $s$.

4. Formula (2.10) should read:

$$
\begin{aligned}
\Omega_{1}(s)= & -\left.(i / \tau) U_{A}^{*}(s) \widetilde{P^{\prime}}(s) U_{A}(s)\right|_{0} ^{s}+O\left(1 / \tau^{2}\right), \\
\Omega_{2}(s)= & \left.(i / \tau) \int_{0}^{s} U_{A}^{*}(t)\left[P^{\prime}(t), P(t)\right] \widetilde{\left(P^{\prime}\right.}(t) U_{A}(t)-U_{A}(t) \widetilde{P^{\prime}}(0)\right) d t \\
& +O\left(1 / \tau^{2}\right) .
\end{aligned}
$$

\footnotetext{
* We thank K. Jung for his help in preparing this note
} 
5. The last terms in Eqs. (2.12), (2.14) and (2.16) are zero because $P^{\prime}$ and $\tilde{X}$ map range $P$ to range $Q:=1-P$ and vice versa. The formulas read

$$
\begin{aligned}
\int_{0}^{t} Q U_{A}^{*}(s) X(s) U_{A}(s) P Y(s) d s= & (i / \tau)\left[\left.Q U_{A}^{*}(s) \tilde{X}(s) U_{A}(s) P Y(s)\right|_{0} ^{t}\right. \\
& -\int_{0}^{t} Q U_{A}^{*}(s) \tilde{X}^{\prime}(s) U_{A}(s) P Y(s) d s \\
& \left.-\int_{0}^{t} Q U_{A}^{*}(s) \tilde{X}(s) U_{A}(s) P Y^{\prime}(s) d s\right], \\
Q(s) X(s) P(s)= & -Q(s)\left[H_{A}(s), \tilde{X}(s)\right] P(s), \\
Q U_{A}^{*}(s) X(s) U_{A}(s) P= & (i / \tau) Q\left(U_{A}^{*}(s) \tilde{X}(s) U_{A}(s)\right. \\
& \left.+U_{A}^{*}(s) \tilde{X}(s) U_{A}^{\prime}(s)\right) P \\
= & (i / \tau)\left[Q\left(U_{A}^{*}(s) \tilde{X}(s) U_{A}(s)\right)^{\prime} P\right. \\
& \left.-Q U_{A}^{*}(s) \tilde{X}^{\prime}(s) U_{A}(s) P\right] .
\end{aligned}
$$

6. The proof of Lemma 2.7 is wrong. It is corrected in the appendix of [2]:

1. Avron, J.E., Seiler, R., Yaffe, L.G.: Adiabatic theorems and applications to the quantum Hall effect. Commun. Math. Phys. 110, 33-49 (1987)

2. Klein, M., Seiler, R.: Power-law corrections to the Kubo formula vanish in quantum Hall systems. Commun. Math. Phys. 128, 141-160 (1990)

Communicated by A. Jaffe 